\title{
Congenital Aneurysm/Diverticulum of the Left Ventricle in the Newborn
}

\author{
Oleksandra Tsaruk $\mathbb{D}^{*}$, Valerian Ryzhyk, Khrystyna Ilnytska(D)
}

\begin{abstract}
Left ventricular aneurysms and diverticula are severe congenital heart defects. The low prevalence and mostly asymptomatic course of these anomalies do not always allow us to suspect these defects in time and to differentiate them among themselves. That is why early detection of pathology with the help of fetal echocardiography is a key factor in determination of the tactics of patients' management. The paper reviews the literature and describes a complex case of prenatally diagnosed left ventricular aneurysm.
\end{abstract}

\section{Keywords}

Left Ventricular Aneurysm; Left Ventricular Diverticulum; Congenital Heart Disease

Department of Radiology and Radiotherapy, Ivano-Frankivsk National Medical University, Ukraine

${ }^{*}$ Corresponding author: medicineforyou@ukr.net

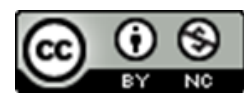

Copyright @Oleksandra Tsaruk, Valerian Ryzhyk, Khrystyna Ilnytska, 2020

\section{Background}

Congenital aneurysms and diverticula of the left ventricle are rare abnormalities in the development of the heart, which are characterized by the presence of local bulging and thinning of the heart wall. It is deemed, that these diseases occur with a frequency of approximately 1 per 200000 liveborn infants. However, some authors note that the true prevalence of these pathologies is unknown, taking into account the possibility of their asymptomic course [7]. In general, over all the time of study of the aneurysms and diverticula of the left ventricle, since their first examination in 1816, there were described less than 1000 cases in the literature [1]. That is why, despite modern advances in echocardiography and radiocardiology, the differential diagnostics between the aneurysm and diverticulum is a demanding task.

Currently, there are several criteria that allow us to distinguish between a diverticulum and aneurysm.

The etiological factor of diverticulum is the delay in the formation of the left ventricular wall in the embryonic period [2]. At the same time, its wall in $87.5 \%$ of cases is represented by all layers of the myocardium and only in $10 \%$ of cases there are fibrous changes $[4,11]$. The contractile ability of the diverticulum is preserved, synchronous. It is characterized by a small size and narrow neck. Some authors note that in some cases the size of diverticulum may remain constant or decrease altogether. The diverticulum is often combined with other congenital malformations, such as abnormalities of the sternum, pericardium, diaphragm, heart (defects of the interventricular and atrial septum) [7, 9]. One of the most severe manifestations of this association is the Pentalogy of Cantrell a group of thoracoabdominal wall defects with cardiac ectopia and omphalocele $[1,2]$.

The substrate for the occurrence of aneurysm is the local myocardial ischemia during embryogenesis [1]. In $89 \%$ of cases, the wall of the anomaly consists of connective tissue, in $11 \%$ of cases all layers of the myocardium and pericardium are preserved [4]. This leads to severe hypo- or akinesia of the wall and asynchrony of contraction. The aneurysm has a broad base, usually larger than the diverticulum. In addition, its size can potentially increase, requiring special care for patients with this pathology [10]. Usually this malformation is isolated - extracardiac abnormalities are observed only in $3.1 \%$ of patients.

The above-mentioned criteria are not always sufficient for the differentiation of these two anomalies. Left ventricular bulging, which simultaneously meet both the criteria of diverticulum and aneurysm, are commonly met in physician's work. Literature sources often describe cases, when the same defect is treated by some authors as a diverticulum, and by some others - as an aneurysm. Such discrepancies arise for a number of reasons. It is noted that the terms such as "narrow neck" and "wide neck" are not objective, and the size of the bulging may change during pregnancy [5]. Contradictions arise not 
only due to the communication problems between clinicians. Insufficient data on the natural course and long-term prognosis of these defects, as well as their significant polymorphism, make it difficult to determine uniform common diagnostic criteria that would reliably help the doctor in determination of the treatment tactics [3].

That is why E. Malakan Rad and co-authors [5] offered a classification of congenital bulges, which is based on the geometry of the left ventricle. The shape of the left ventricle, the thickness and regional contractility of the bulging wall are of great importance in this new classification:

- I type - the left ventricle has a shape disorder, but the thickness and regional contractility of the defect wall are preserved.

- IIa type - the left ventricle has a violation of shape, the wall thickness of the defect is reduced.

- IIb type - the left ventricle has a violation of shape, the regional contractility of the defect wall is reduced.

- IIc type - the left ventricle has a violation of shape, wall thickness and regional contractility of the defect wall are reduced.

The authors have also created a system for the estimation of the congenital bulges' size, which is based on the ratio of echocardiographic parameters of the defect and the left ventricle:

Index of congenital bulge area $=$ $\frac{\text { left ventricular area }}{\text { congenital bulge area }}$

Index of congenital bulge circumference $=$ $\frac{\text { left ventricular circumference }}{\text { congenital bulge circumference }}$

Index of congenital bulge volume $=$ $\frac{\text { left ventricular volume }}{\text { congenital bulge volume }}$

The bulge is considered small if the circumference index is less than $1 / 3$. It is considered medium if the area index is greater than $1 / 3$, but less than $1 / 2$. And it is considered large if the volume index is greater than $1 / 2$ [5]. Thus, this classification allows us to complement the diagnostic criteria with objective echocardiographic data.

Echocardiography is a generally available, non-invasive and highly-informative method of diagnostics of the congenital aneurysms and left ventricular diverticula. It allows us to accurately assess the size of the bulge, the contractility of the defect wall and left ventricle, the presence of other congenital heart defects.
These defects can cause a wide range of different symptoms, although in $42 \%$ of patients with aneurysms and in $63 \%$ of patients with diverticula, the course is asymptomatic. Severe complications such as bulging rupture, thrombosis, or embolism have developed in only in $4 \%$ of patients aged less than 18 years old [4]. In more than half of the cases, these defects are diagnosed accidentally during the echocardiography, multispiral computed tomography, or magnetic resonance imaging. Often diverticula and aneurysms of the left ventricle are the findings in the diagnosis of various genetic pathologies.

Possibilities of conservative treatment, indications for surgical interventions and terms of their performance remain debatable [12]. The technique of surgical correction of the defect is determined by the size of the bulge and the final diastolic volume of the left ventricle [1].

The prognosis and long-term consequences vary greatly depending on the bulge type. Based on the long-term observation, it was found that within 8.5 months since birth, $40 \%$ of children with left ventricular aneurysm have developed complications. In contrast, during the ten years' observation of children with diverticula, there were no serious complications [3]. In general, the prognosis for these defects is good, in many children the disease is asymptomatic for a long time, although there is a risk of the development of severe complications such as aneurysm or diverticulum rupture, arrhythmias, thromboembolism, pericardial effusion and death $[4,6,8]$.

\section{Case Presentation}

Heart defect is diagnosed according to prenatal ultrasound diagnostics. Echocardiography of the fetus (21.05.20): Pregnancy 1 with live fetus, $34-35$ weeks. The fetus has a heart defect: Aneurysm of the apex of the left ventricle with akinesia. The total systolic contractility of the left ventricle is preserved. There is a minimal mitral valve insufficiency. There is the only artery of the umbilical cord.

Girl A., born on July 1, 2020, was born as a result of the second pregnancy (gestational age 38 weeks), the second childbirth. Apgar score 7 - 8. The pregnancy took place on the background of mild anemia.Childbirth were performed by cesarean section. Birth weight $-3260 \mathrm{~g}$, body length $54 \mathrm{~cm}$. Blood group and rhesus of mother A (II)+, infant's A (II)+. After birth, the newborn was immediately transferred to the neonatal intensive care unit of the Ivano-Frankivsk Regional Perinatal Center to confirm the diagnosis and determine further therapeutic tactics.

The newborn's condition was considered serious due to congenital heart disease. Body temperature - was $36.8^{\circ} \mathrm{C}$. Skin cover: acrocyanosis. Mucous membranes were moist, pale pink, without rashes. Turgor and skin elasticity were preserved. There was pastosity of the legs. Frequency of respiratory movements $-50 / \mathrm{min}$, rhythmic breathing, without the participation of auxiliary muscles. Auscultation was performed evenly, weakened. Respiration was independent, oxygen independent, $\mathrm{SpO} 2-94 \%$.

Heart tones were rhythmic, muffled, systolic murmur 
along the left edge of the sternum. Heart rate - was 134/min., average blood pressure $-55 \mathrm{~mm} \mathrm{Hg}$. Pulsation on peripheral arteries was clear, pulse of satisfactory filling. The abdomen was soft. The liver was palpated along the edge of the costal arch.

Neurosonography (02.07.20): No displacements of the median structures are detected. III ventricle is not enlarged. IV ventricle was not enlarged. Interhemispheric gap is 3 $\mathrm{mm}$. Convexital spaces are of $3 \mathrm{~mm}$. Cavity of a transparent membrane is $3 \mathrm{~mm}$. Large cistern is $6 \mathrm{~mm}$. Brain parenchyma is of medium echogenicity. Periventricular areas - of increased echogenicity. Periventricular edema is of the I-II degree. There is immaturity of brain structures.

Echocardioscopy (02.07.20): Heart chambers are not enlarged. The walls of the myocardium are not thickened. The general contractile ability is good. Emission fraction $-60 \%$. Functioning oval window is $6.5 \mathrm{~mm}$, discharge is leftright. A round aneurysm, diameter $1.52 \mathrm{~cm}$, volume $1.46 \mathrm{~cm}^{3}$, is visualized in the projection of the apex (Fig. 1).

Ultrasound examination of the abdominal cavity (02.07.20) The liver protrudes $1 \mathrm{~cm}$ from the edge of the costal arch. Gallbladder - is of the oval shape. The pancreas is within normal limits. Spleen - without peculiarities. Kidneys - the ratio of parenchyma to the middle complexes is preserved. No free fluid was detected. Adrenal glands - the structure is preserved.

The newborn was consulted by a cardiologist (02.07.20). Diagnosis: Aneurysm of the apex of the left ventricle. Functioning oval window $(6.5 \mathrm{~mm}$.). Mitral valve insufficiency. A consultation at the Scientific-Practical Medical Center of Pediatric Cardiology and Cardiac Surgery is recommended.

Echocardioscopy (22.07.20): The chambers of the heart are not enlarged. The walls of the myocardium are not thickened. The general contractile ability is good. Emission fraction $-67 \%$. Functioning oval window, without overloading the left departments of the heart. Aneurysmal dilatation with a volume of $1.9 \mathrm{~cm}^{3}$ with myocardial thinning up to $2.1-$ $2.2 \mathrm{~mm}$, is visualized in the apex projection.

Electrocardiography (22.07.20): Sinus rhythm, heart rate $158 / \mathrm{min}$. The electrical axis of the heart is deflected to the right. There is the deviation of repolarization processes (elevation of ST segment).

At the age of 1 month (30.07.20) the newborn was consulted in the Scientific-Practical Medical Center of Pediatric Cardiology and Cardiac Surgery. Echocardioscopy was performed (30.07.20): Aneurysm of the anterior-apical part of the left ventricle. The interventricular septum in the area of the aneurysm is thinned up to $1.5-1.6 \mathrm{~mm}$. Teicholz emission fraction $-68 \%$. According to Simpson, the final diastolic volume is $11.5 \mathrm{ml}$, the final systolic volume is $36 \mathrm{ml}$, and the ejection fraction is $56 \%$. The total contractility is preserved with hypokinesis in the area of the left ventricular aneurysm. There was made the diagnosis: Aneurysm of the apex of the left ventricle with hypokinesis of the myocardial wall. The total contractility is preserved, with hypokinesis in the area of the left ventricular aneurysm. Minimum open
Figure 1. Case of left ventricular aneurysm.

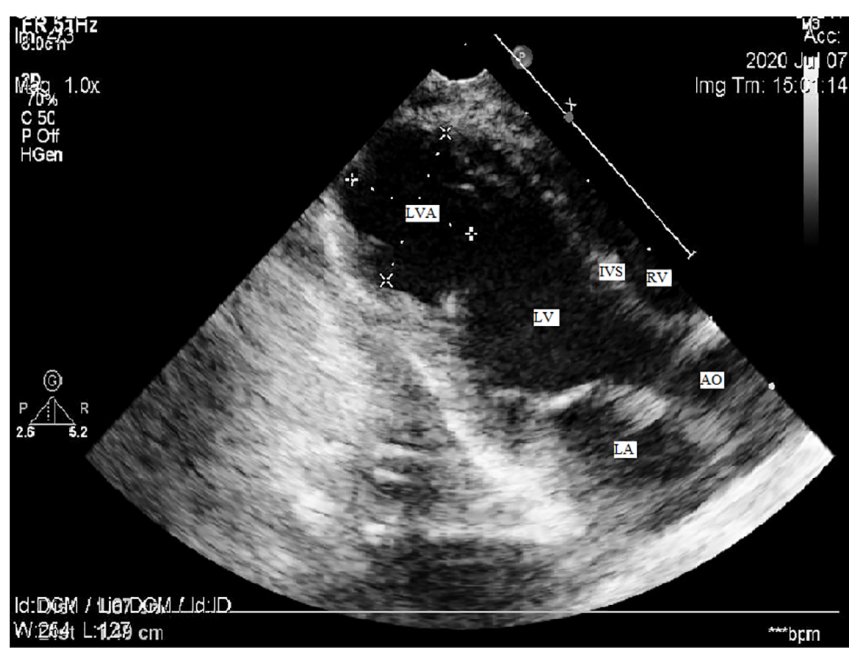

(a) Ultrasound section - large aneurysm with a broad connection to left ventricle. LA: left atrium; RV: right ventricle; $L V$ : left ventricle; AO: aorta; IVS: interventricular septum; LVA: left ventricular aneurysm.

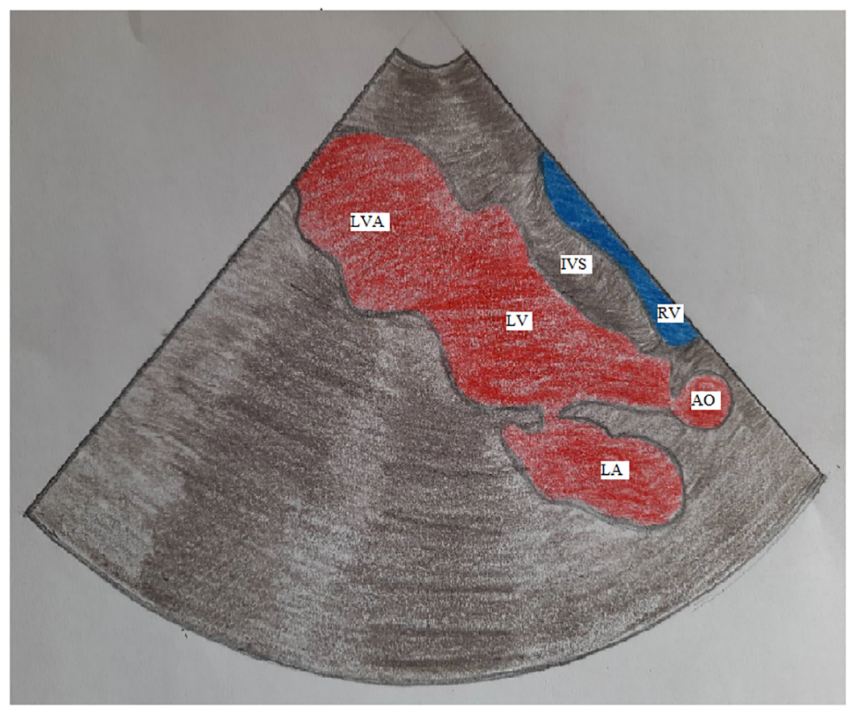

(b) Diagrammatical representation.

oval window. The cardiologist's observation at the place of residence, electrocardiography and echocardioscopy - after 2 months, scheduled re-consultation were recommended.

Echocardioscopy in dynamics (06.10.20): The chambers of the heart are not enlarged. The walls of the myocardium are not thickened. The general contractile ability is good. Emission fraction - is $65 \%$. There is functioning oval window, without overloading of the left departments of the heart. Aneurysmal dilatation with a volume of $2 \mathrm{~cm}^{3}$ with myocardial thinning up to $2.1-2.7 \mathrm{~mm}$, is visualized in the apex projection. There is no dynamics from the previous examination. 
Electrocardiography in dynamics $(06.10 .20)$ : Sinus rhythm, heart rate 136/min. The electrical axis of the heart is vertical. Deviation of repolarization processes (positive dynamics).

Nowadays, the follow-up of the patient is 4 months. Mother has no complaints about the child's health. Physical development is harmonious. Psycho-motor development is according to age. Echocardiographic picture is without negative dynamics. There are no signs of decompensation of congenital heart disease. It is recommended to perform reexamination of the child at the age of 5 months.

\section{Conclusions}

Congenital aneurysms and diverticula of the left ventricle are very rare, potentially fatal abnormalities. Differential diagnostics between these defects is extremely important, because they have different course, treatment and prognosis. Since the clinical picture does not allow us to reliably distinguish these anomalies, the main key to an accurate diagnosis is awareness of diagnostic criteria and timely detection of this pathology. Therefore, fetal echocardiography in recent years is an important tool for early prenatal diagnosis, and as a consequence, contributes to the effective management of children with such defects. Our clinical case shows, that the early detection of the defect in the period of 34-35 weeks of pregnancy allowed us in the shortest possible time send patient for dynamic monitoring to a specialized institution.

\section{Informed Consent}

The mother has signed an informed voluntary consent to examine her child.

\section{Conflict of Interest}

The authors declare that no conflicts exist.

\section{Financial Disclosure}

The authors declared no financial support.

\section{References}

[1] Soynov IA, Gorbatykh YN, Kulyabin YY, Kornilov IA, Nichay NR, et al. Diverticula and Congenital Aneurysms of the Left Ventricle: Anatomical Features, Pathophysiology, Clinical Presentation and Treatment Strategy. Kardiologiia [Internet]. 2018;17(2):33-39. Available from: https://doi.org/10.18087/cardio.2018.2.10066

[2] Halpern L, Garabedian C, Worrall NK. Congenital Ventricular Diverticulum or Aneurysm: A Difficult Diagnosis to Make. Case Reports in Cardiology [Internet]. 2018 Nov 11;2018:1-5. Available from: https://doi.org/10.1155/2018/5839432

[3] Ohlow M-A, Brunelli M, Lauer B. Characteristics and outcome of primary congenital left ventricular aneurysm and diverticulum: analysis of cases from the literature. Prenatal Diagnosis [Internet]. 2014 May 15;34(9):893-899. Available from: https://doi.org/10.1002/pd.4389

[4] Ohlow M-A, von Korn H, Lauer B. Characteristics and outcome of congenital left ventricular aneurysm and diverticulum: Analysis of 809 cases published since 1816. International Journal of Cardiology [Internet]. 2015 Apr;185:34-45. Available from: https://doi.org/10.1016/j.ijcard.2015.03.050

[5] Malakan Rad E, Awad S, Hijazi ZM. Congenital Left Ventricular Outpouchings: A Systematic Review of 839 Cases and Introduction of a Novel Classification after Two Centuries. Congenital Heart Disease [Internet]. 2014 Aug 27;9(6):498-511. Available from: https://doi.org/10.1111/chd.12214

[6] Hirose A, Maeno Y, Suda K, Fusazaki N, Kado H, Matsuishi T. Serial hemodynamic assessment using Doppler echocardiography in a fetus with left ventricular aneurysm presented as fetal hydrops. Journal of Perinatology [Internet]. 2013 May 30;33(6):486-489. Available from: https://doi.org/10.1038/jp.2012.93

[7] Gowda M, Bharathi S, Thiagarajan M, Aneja T. Prenatal diagnosis of fetal right and left congenital ventricular aneurysms. The Journal of Maternal-Fetal \& Neonatal Medicine [Internet]. 2017 Jun 28;31(17):2367-2370. Available from: https://doi.org/10.1080/14767058.2017.1342799

[8] Shuplock JM, Kavanaugh-McHugh A, Parra D. Prenatally Diagnosed Congenital Ventricular Outpouchings: An Institutional Experience and $\mathrm{Re}$ view of the Literature. Pediatric Cardiology [Internet]. 2019 Nov 14;41(2):272-281. Available from: https://doi.org/10.1007/s00246-019-02252-7

[9] Ohlow MA. Congenital left ventricular aneurysms and diverticula: an entity in search of an identity. J Geriatr Cardiol. 2017 Dec;14(12):750-762. Available from: https://www.ncbi.nlm.nih.gov/pmc/articles/PMC5863053/

[10] Umapathi KK, Bokowski JW, Nguyen HH. Congenital right ventricular aneurysm with characteristics of a pseudoaneurysm. Cardiology in the Young [Internet]. 2020 Mar 27;30(5):732-733. Available from: https://doi.org/10.1017/S1047951120000633

[11] Marchesi M, Boracchi M, Gentile G, Maghin F, Zoja R. Sudden perinatal death due to rupture of congenital cardiac diverticulum. Pathological findings and medico-legal investigations in malpractice charge. Legal Medicine [Internet]. 2017 Sep;28:6-9. Available from: https://doi.org/10.1016/j.legalmed.2017.07.003 
[12] Hofbeck M, Sieverding L, Grözinger G, Kagan KO. Coil occlusion of a congenital left ventricular aneurysm in a newborn. Catheterization and Cardiovascular Interventions [Internet]. 2020 Mar 19;96(2):389-392. Available from: https://doi.org/10.1002/ccd.28849
Received: 2020-10-15

Revised: 2020-10-29

Accepted: $2020-11-16$ 